\title{
Editorial
}

\section{Special feature on AKARI results}

\author{
C. Bertout
}

Editor-in-Chief, Astronomy \& Astrophysics, Observatoire de Paris, 61 Av. de l'Observatoire, 75014 Paris, France

The Japanese astronomical satellite operated in collaboration with ESA, AKARI, was launched in February 2006 and it performed an all-sky survey in the mid-infrared $(9$ and $18 \mu \mathrm{m})$ and far-infrared $(65,90,140,160 \mu \mathrm{m})$ over 16 months from May 2006 to August 2007. AKARI also performed pointed observations, providing comprehensive multi-wavelength photometric and spectroscopic coverage of a wide variety of astronomical sources.

On the occasion of the first public release of the all-sky survey point source catalogs, we present a compendium of results from AKARI featuring 17 papers ranging from all-sky survey results obtained in the validation phase, deep surveys, and open time results.

Acknowledgements. Many thanks to Alberto Salama, who acted as coordinator for this A\&A special feature. 